\title{
Improving Customer Satisfaction by the Expert System Using Artificial Neural Networks
}

\author{
FENG QIAN \\ Institute of Management Science \& Information \\ Engineering \\ Hangzhou Dianzi University \\ P.R. CHINA \\ qianf@hdu.edu.cn
}

\author{
LINWEN XU \\ Hangzhou Institute of Commerce \\ Zhejiang Gongshang University \\ P.R. CHINA \\ xulw@mail.zjgsu.edu.cn
}

\begin{abstract}
CRM, which aims to enhance the effectiveness and performance of the businesses by improving the customer satisfaction and loyalty, has now become a leading business strategy in highly competitive business environment. The objective of this research is to improve customer satisfaction on product's colors with the aid of the expert system developed by the authors by using artificial neural networks. The expert system's role is to capture the knowledge of the experts and the data from the customer requirements, and then, process the collected data and form the appropriate rules for choosing product's colors. In order to identify the hidden pattern of the customer's needs, the artificial neural networks technique has been applied to classify the colors based upon a list of selected information. Moreover, the expert system has the capability to make decisions in ranking the scores of the colors presented in the selection. In addition, the expert system has been validated with a variety of customer types.
\end{abstract}

Key-Words: - Expert system; Artificial neural networks; Customer relationship management; Back propagation; Customer satisfaction; Decision support

\section{Introduction}

The core part of CRM activities is to understand customer requirements and retain profitable customers. To reach it in a highly competitive market, satisfying customer's needs is the key to business success [1]. However, it is a difficult and a complex task to identify the customer's needs such as colors of the products. The objective of this research, therefore, is to design and implement the expert system in order to assess customer satisfaction and reveal appropriate strategies to improve it. As the customer satisfaction on colors can have a complex hidden pattern and, therefore, the approach of the research should have an ability to perform pattern recognition, classification and forecast which make the artificial neural networks an appropriate technique to be applied in the expert system. The concept of the research is illustrated in Fig. 1, in which the assumption of the customer requirements and expert system is based upon the statement that "in general, the same customer group will like the same colors".

The organization of the paper is the following: First, in Section 2, we give a short introduction to expert system and artificial neural networks, with some relevant literatures. Section 3 describes the proposed research work in relation to. We present the designing of our expert system, especially the artificial neural networks. After presenting our approach, we discuss in Section 4 the results of an experiment we performed to validation the system. Finally, in Section 5, is the concluding.

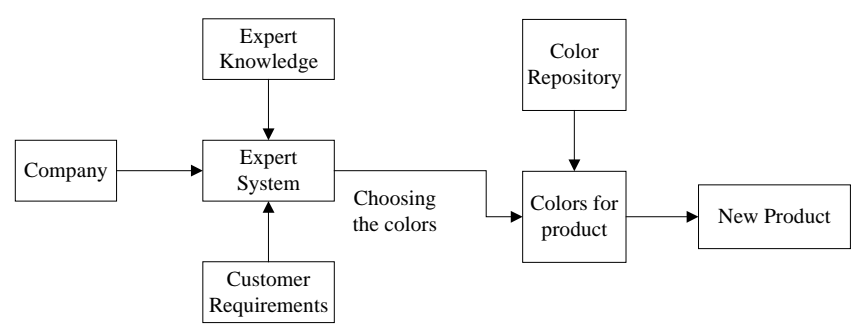

Fig. 1 The conceptual of the research.

\section{Expert system and artificial neural networks}

\subsection{Expert System}


An expert system is the computer system that emulates the behavior of human experts in a well-specified manner, and narrowly defines the domain of knowledge. It captures the knowledge and heuristics that an expert employs in a specific task [2]. An overview of current technologies applied with an expert system that is developed for Database Management System, Decision Support System, and the other intelligent systems such as Neural Networks System, Genetic Algorithm, etc., can be seen in [3].

In this research, the expert system roles have been designed to capture the knowledge of the experts and the data of customer requirements. After that the system is able to process these data and form rules to customize colors in products. Finally, the expert system will deliver the decision ranking of the scores based upon the product samples, which are presented in the selection process.

\subsection{Artificial Neural Networks}

Artificial Neural Networks (ANNs) are generic non-linear function approximations extensively used for pattern recognition and classification [4]. An ANN is a collection of basic units, called neurons, computing a non-linear function of their input. Every input has an assigned weight that determines the impact this input has on the overall output of the node. In Fig. 2 it is possible to see a schematic representation of such an artificial neuron, where $w_{j i}$ is the weight of the connection from neuron $i$ to neuron $j$, and $o_{j}$ is the activation or output of neuron $j$. Unit $j$ output is obtained by ideally following a two step-procedure. First the total weighted input $z_{j}$ is computed using the formula $z_{j}=\sum_{i} w_{j i} o_{i}$ where $o_{i}$ is the activity level of the $i$-th unit in the previous layer and $w_{j i}$ is the weight of the connection between the $i$-th and the $j$-th unit. Then, the neuron output is obtained as a non-linear function, shown in fig. 2.

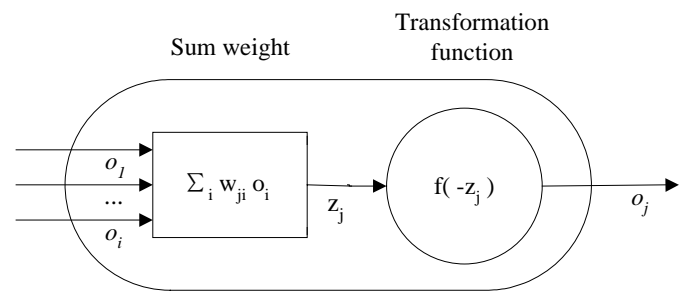

Fig. 2 Artificial neuron $j$.

By interconnecting a proper number of nodes in a suitable way and by setting the weights to appropriate values, ANN can approximate any non-linear function with arbitrary precision [5]. This structure of nodes and connections, known as network topology, together with the weights of the connections, determines the final behavior of the network. Given ANN topology and a training set, it is possible to optimize the values of the weights in order to minimize an error function by means of any back propagation algorithm, standard optimization techniques or randomized algorithms.

In practical terms, a product may have several colors mixed together. Based upon this complicated situation, we need to apply the artificial intelligence system by using ANN to classify the colors that are appropriate to the consumer requirements. In this research, we use the multilayer architecture, back propagation algorithm [6], in a supervised learning method, to classify the colors that are preferred by consumers.

\section{Proposed research work}

In order to develop the expert system, some steps, which consist of gathering the raw data, system designing, programming and testing, verifying and validating, and evaluation must be fulfilled.

In this section, we describe only system designing. Some other steps are explained together with the experimentation in Section 4.

\subsection{The Expert System Designing}

Based upon Medsker and Liebowitz concerning the theory of expert systems [2], which should have three main components that are:

- User interface.

- Inference engine (for making decision).

- Database (for storing the data, rules, and training the system).

- Fig. 3 illustrates the system model that embraces the ANN in the expert system. The ANN is preferred in this research because its ability to clarify and classify complex hidden pattern of the customer requirements and to forecast colors that can satisfy different types of customer.

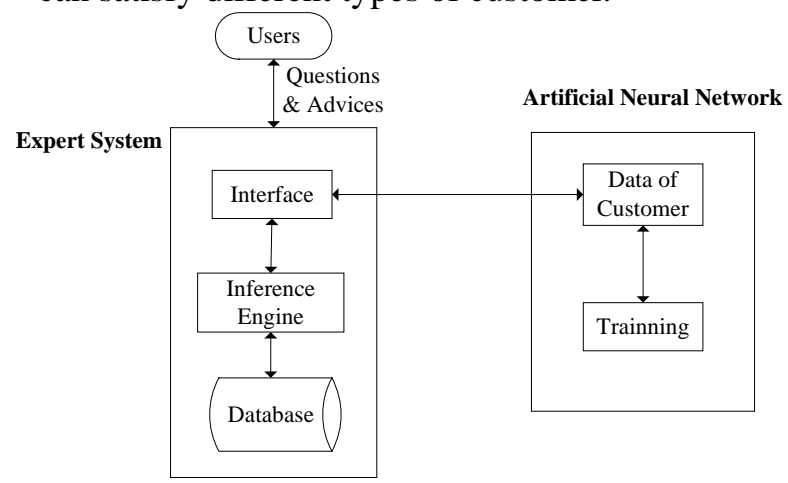

Fig. 3 The expert system model. 
The customers (users) can interact with the interface of the expert system to ask and get the advices from the system. The Inference engine consists of two algorithms:

- The production rules from expert knowledge and

- The data of customers that are classified by the ANN system.

\subsection{The Artificial Neural Networks Designing}

Medsker and Liebowitz [2] mention that the common multilayer architecture is more appropriate for classification applications, and this is the reason we select multilayer architecture to classify colors in this research. They recommend including eight input nodes (which means eight types of customer) in the first layer. The second layer (called hidden layer) should have 30 nodes. The third layer (called the output layer) should have 52 nodes (which means 52 types of mixed colors). So, the total multilayer architecture in this research has 8 input, 30 hidden, and 52 output nodes.

We choose the mathematical model at a neuron as shown in Fig. 2. Considering the task to train the data of customer requirements, we apply the multilayer architecture with back propagation learning rule [3] by adjusting the weight matrix. The structure of the ANN is illustrated in Fig. 4.

The model has the mathematic functions as below, and the model parameters are illustrated in Table 1.

- Transformation function:

$o_{j}=f\left(z_{j}\right)=\frac{1}{1+\exp \left(-z_{j}\right)} \quad z_{j}=\sum_{i} w_{j i} o_{i}$

- The error equation:

$e_{j}(n)=d_{j}(n)-o_{j}(n)$

- Slope at output layer:

$\delta_{j}^{(L)}(n)=e_{j}(n) o_{j}(n)\left[1-o_{j}(n)\right]$

- Slope at the other layer:

$\delta_{j}^{(l)}(n)=y_{j}(n)\left[1-y_{j}(n)\right] \sum_{k} \delta_{k}^{(l+1)}(n) w_{k j}^{(l+1)}(n)$

- Weight update:

$$
\begin{aligned}
w_{j i}^{(l)}(n+1)=w_{j i}^{(l)}(n)+ & \alpha\left[w_{j i}^{(l)}(n)-w_{j i}^{(l)}(n-1)\right] \\
& +\eta \delta_{j}^{(l)}(n) y_{j}^{(l-1)}(n)
\end{aligned}
$$

- Learning rate $(\alpha)=0.2$ (according to [3]).

- Momentum constant $(\eta)=0.5$ (according to [3]).

From Eq. (1) we can illustrate the ANN computation as follows.

Input layer: The input signal in each node is " 1 " and the node that has no signal is " 0 ".

Hidden layer: At a node, the input values by the weights are sum to this node and transfer to next layer by the Eq.(1).

Table 1 Model parameters

\begin{tabular}{ll}
$y:$ Output at a layer & $L:$ Output layer \\
$d:$ Desired output & $l:$ First and second (hidden) layer \\
$e:$ Error & $n:$ Learning iteration (epoch) \\
$o:$ Computation output & $\delta_{j}:$ Slope at a layer \\
$\alpha:$ Learning rate & $w_{j i}:$ Weight matrix \\
$\eta:$ Momentum constant & $\delta_{k}:$ Previous slope (back \\
& propagate) \\
\hline
\end{tabular}

From Eqs.(2) to (5) when we try to train the system, the weight matrix will be changed, and the networks computation will classify the data more precisely than the previous computation. It has been computed in order to achieve the result and the error can be computed by Eq.(2).

Output layer: Then, the error values are propagated to the previous layer to adjust the weight matrix. At first we calculate the slope at a layer by the Eq. (3). The weights updated are computed by the Eq. (5).

Hidden and input layer: The hidden and input layer use similar computing, but the Eq. (4) is substitute to the Eq. (3). Ultimately, the hidden layer can precisely transfer the data from the output layer to the first layer.

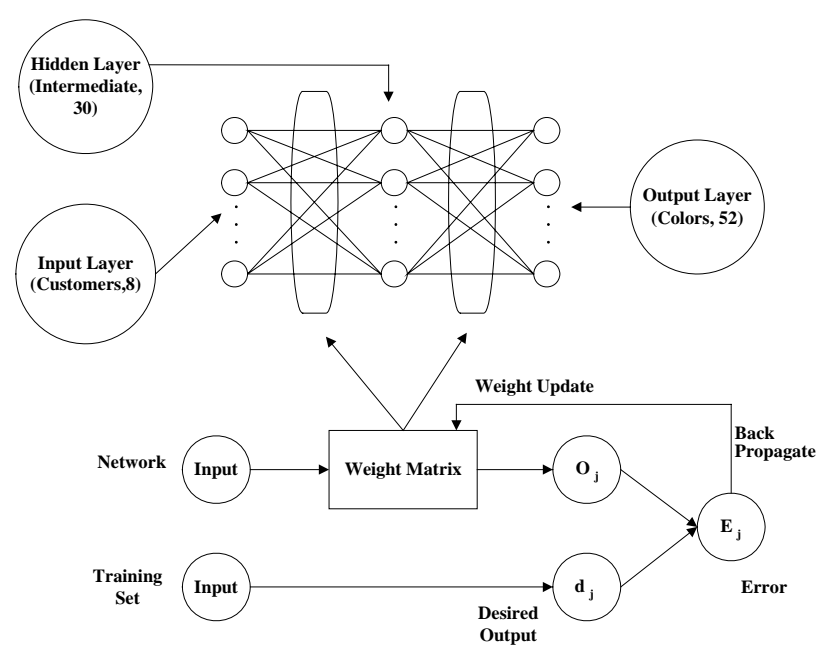

Fig. 4 The multilayer architecture with back propagation.

\section{Discussion of experimentation}

Having designed the expert system including ANN, we implemented it and initially tested it by the simulated data. We input the sample data of the expert knowledge and the data of customer requirements. The results demonstrated that the system was working correctly 
and was ready to be applied to the real case.

As we were looking to evaluate our research on a real world case, we established collaboration with a manufacture producing mp3. Its aim is to produce a new mp3 for young male / female as target groups. The firm needs to select some colors from their repository based upon the ability of the expert system. Our aim is to watch the chosen colors advised by the system, for each type of customer.

According to [2], the evaluating data should consist of 16 cases as follows:

- 2 decision, by expert knowledge and data of customer requirements,

- 2 genders, male, and female,

- 4 age groups, teen, young, adult, and senior.

\subsection{Gathering the Raw Data}

Gathering the raw data contains expert knowledge acquisition and the customer requirements data.

As there is only limited knowledge from literature, we have to acquire knowledge directly from the designer the products. The acquired knowledge consists of the fundamental principles of using colors, the materials, the techniques, how colors are used in producing, and the criteria for choosing them that are appropriate to the consumers. This means the data in this viewpoint is from the expert and manufacturer side.

The customer requirements data, as mentioned earlier, the first layer, the input layer, should have eight nodes, the list below shows eight varieties of customers from the first survey.

- Male-teen (13-18 years old)

- Male-young (19-29 years old)

- Male-adult (30-45 years old)

- Male-senior (over 46 years old)

- Female-teen (13-18 years old)

- Female-young (19-29 years old)

- Female-adult (30-45 years old)

- Female-senior (over 46 years old)

We firstly recorded data from a number of customers who bought $\mathrm{mp} 3$ at the selected IT counters in Hangzhou, Zhejiang, P.R.China. The data containing customers' gender, age, the colors they had selected.

\subsection{Performance Evaluation}

After completing the recording of the expert knowledge and the details of the eight groups of customers (male/female of teen, young, adult, and old) purchased into the expert system on chosen products' characteristics about colors, we evaluate the expert system (including ANN) performance by doing the survey 240 data based upon similar eight groups of customers, (similar in eight group types but different in person). As mentioned earlier, the aim of the survey is to evaluate the accuracy between the result advised by the expert system and the chosen product (colors) from each type of any customer. So, the higher number of customer satisfaction on the product (colors) advised by the expert system means higher accuracy of the expert system. The expert system performance evaluation is illustrated in Table 2, for example, at product sample S6, 58 out of 76 females at a young age chose similar to the result advised from the expert system, which means it can predict precisely at approximate $76.3 \%$. In terms of males at a young age, the expert system predicts with $66.7 \%$ accuracy. Based upon the firm target groups of male/female at young age, the result advised from the expert system (higher than 65\%) can satisfy the firm's management. However, the average percentage of the correctness is $59.4 \%$ that is a moderate to high correct result. The reason is that at mp3 sample S4, male/old age, there are only a few data (four in total) and only one customer prefers the recommended color from the expert system which produces a percent correctness of only $25 \%$. The rest of the correctness percentages are all above $50 \%$.

Table 2 Performance evaluation

\begin{tabular}{|c|c|c|c|c|c|c|c|c|c|}
\hline \multirow{2}{*}{$\begin{array}{l}\text { Product } \\
\text { samples }\end{array}$} & \multicolumn{4}{|l|}{ Male } & \multicolumn{5}{|c|}{ Female } \\
\hline & Teen & Young & Adult & Old & Teen & Young & Adult & Old & Total \\
\hline S1 & 25 & 3 & 1 & 0 & 3 & 1 & 1 & 2 & 36 \\
\hline S2 & 5 & 14 & 1 & 1 & 3 & 1 & 0 & 0 & 25 \\
\hline S3 & 0 & 3 & 4 & 1 & 1 & 2 & 0 & 1 & 12 \\
\hline S4 & 0 & 0 & 1 & 1 & 0 & 0 & 1 & 0 & 3 \\
\hline S5 & 2 & 1 & 0 & 0 & 43 & 10 & 3 & 0 & 59 \\
\hline S6 & 1 & 1 & 0 & 0 & 11 & 58 & 3 & 2 & 76 \\
\hline S7 & 0 & 0 & 1 & 0 & 1 & 2 & 12 & 1 & 17 \\
\hline S8 & 0 & 0 & 0 & 1 & 1 & 2 & 1 & 7 & 12 \\
\hline Total & 33 & 22 & 8 & 4 & 63 & 76 & 21 & 13 & 240 \\
\hline \multicolumn{10}{|l|}{ Conclusion } \\
\hline Samples & S1 & S2 & S3 & S4 & S5 & S6 & S7 & S8 & Average \\
\hline \%Correct & 78.1 & 66.7 & 50.0 & 25.0 & 68.3 & 76.3 & 57.1 & 53.9 & 59.4 \\
\hline
\end{tabular}

\section{Conclusions}

Across market segments, a vast variety of colors mixing in different products that makes it a difficult and complicated task to identify the customer's needs. The contribution of this research is in designing the system that is the combination of the expert system and the ANN. In order to validate the approach, the expert system is put to test with surveys data within the 
market. There are two main results from this research. First, knowledge could be captured not only from the designer, also from data analyzing.

Another main result, knowledge from data analyzing, guides us in detail about how to utilize customer behavior on preferred products (colors) in combination with knowledge captured from the expert in prediction of the colors. This benefits greatly the manufacturer in offering the right colors to the right customer group of its new product in order to achieve customer satisfaction.

In terms of limitations, this system takes time to train, particularly if there are a lot of different colors (data). This is because we design the ANN by using a "supervised learning method" that allows a more accurate and appropriate result with a large scale of data. If the amount of data is small, we may design the ANN by using an "unsupervised learning method" which uses a different set of equations. Another limitation is that this research initially aims to assess customer satisfaction on colors, therefore, it could be appropriate to the mp3 industry although the model itself is feasible and applicable to some other type of business, for example, food and beverages, some sensory factors in products, etc. It can also be applied in other intelligence machines such as electronic nose, perfume mixer machine and gas chromatography, etc.
References:

[1] A. Kengpol, "Using information quality techniques to improve production planning and control," International Journal of Management, 23(1), 2006, pp. 53-60.

[2] L. Medsker, and J. Liebowitz, Design and development of expert systems and neural networks, NY, USA: McMillan College Publishing Company, 1994.

[3] E. Turban, and J. E. Aronson, Decision support systems and intelligent system,. NJ, USA: Prentice-Hall, Inc., 2001.

[4] S. Haykin, Neural Networks, A Comprehensive Foundation, $2^{\text {nd }}$ ed. NJ, USA: Prentice Hall, Inc., 1999.

[5] K. M. Hornik, M. Stinchcombe, and H. White, "Multilayer feed forward networks are universal approximators," Neural Networks 2, 1989, pp. 359-366.

[6] M. T. Hagen, H. B. Demuth, and M. Beale, Neural Network Design, Boston, USA: PWS Publishing Company, 1996. 